\title{
SIRT1 Alleviates Aldosterone-Induced Podocyte Injury by Suppressing Mitochondrial Dysfunction and NLRP3 Inflammasome Activation
}

\author{
Mingzhu Jiang ${ }^{a, b}$ Min Zhao ${ }^{b, c}$ Mi Baia,b Juan Lei ${ }^{a, b} \quad$ Yanggang Yuand \\ Songming Huang ${ }^{a, b}$ Yue Zhang ${ }^{a, b}$ Guixia Dinga,b Zhanjun Jia ${ }^{a, b}$ \\ Aihua Zhang a, b \\ ${ }^{a}$ Department of Nephrology, Children's Hospital of Nanjing Medical University, Nanjing, China; ${ }^{b} J i a n g s u$ Key \\ Laboratory of Pediatrics, Nanjing Medical University, Nanjing, China; 'Department of Nephrology, Nanjing Drum

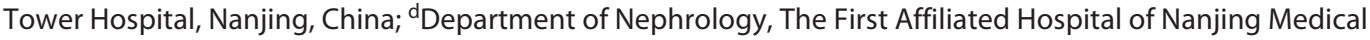 \\ University, Jiangsu Province Hospital, Nanjing, China
}

\section{Keywords}

Silent mating type information regulation 2 homolog 1 . NLR family pyrin domain containing 3 inflammasome . Mitochondrial dysfunction - Aldosterone - Podocyte injury

\begin{abstract}
Background: Podocyte injury contributes to progressive glomerulosclerosis. Previously, we demonstrated the important role of the NLR family pyrin domain containing 3 (NLRP3) inflammasome in mediating the podocyte injury induced by aldosterone. Silent mating type information regulation 2 homolog 1 (SIRT1) is an NAD+-dependent deacetylase that is associated with the regulation of cellular inflammation. However, whether the activation of the NLRP3 inflammasome in podocytes is regulated by SIRT1, and the mechanism involved, remains unknown. Methods: In this study, we detected SIRT1 expression in patients with podocyte disease and performed an aldosterone infusion model in podocytespecific Sirt1 knockout mice. In cultured podocytes, we used plasmids to overexpress SIRT1 and treated the podocyte with aldosterone. Results: SIRT1 was significantly decreased in the glomeruli of patients with podocyte disease. Sirt1-de-
\end{abstract}

karger@karger.com www.karger.com/kdd

Karger $\stackrel{\text { ! }}{\div}$

GOPEN ACCESS
(C) 2021 The Author(s)

Published by S. Karger AG, Basel

This is an Open Access article licensed under the Creative Commons Attribution-NonCommercial-4.0 International License (CC BY-NC) (http://www.karger.com/Services/OpenAccessLicense), applicable to the online version of the article only. Usage and distribution for commercial purposes requires written permission. ficient mice showed significant urinary albumin excretion after aldosterone infusion, and the severity of the glomerular injury was significantly greater in podocyte-specific Sirt1 knockout mice than in the wild-type mice. Moreover, podocyte conditional Sirt1 knockout aggravated NLRP3 inflammasome activation and mitochondrial dysfunction (MtD). In vitro, overexpression of SIRT1 inhibited NLRP3 activation, protected against MtD and podocyte injury. Conclusion: Taken together, these findings revealed a novel regulatory mechanism of the NLRP3 inflammasome by SIRT1 by promoting mitochondrial function, which provides some potential targets for the treatment of glomerular diseases.

(c) 2021 The Author(s)

Published by S. Karger AG, Basel

\section{Introduction}

Podocytes are terminally differentiated and highly specialized cells. Podocyte injury is a key component of progressive glomerulosclerosis. Growing evidence sug-

Mingzhu Jiang and Min Zhao have contributed equally to this work.
Aihua Zhang

Department of Nephrology, Nanjing Children's Hospital

Affiliated to Nanjing Medical University

72 Guangzhou Road, Nanjing 210029 (China)

zhaihua@ njmu.edu.cn 
gests that podocyte injury leads to proteinuria and aggravated progressive glomerulosclerosis in many glomerular diseases [1].

Mitochondria are essential intracellular organelles for energy production via ATP synthesis using oxidative phosphorylation and play a key role in apoptosis [2]. Recently, studies have shown that mitochondrial dysfunction (MtD) is associated with many types of kidney disease. $\mathrm{MtD}$ is evidenced by overproduction of reactive oxygen species (ROS) in mitochondria, mitochondrial DNA (mtDNA) damage, and respiratory chain dysfunction [3]. We also demonstrated that MtD mediates aldosteroneinduced podocyte injury $[4,5]$.

NLR family pyrin domain containing 3 (NLRP3) is a member of the NLR family of intracellular pattern recognition receptors that participate in nonmicrobial inflammation. ROS derived from an uncharacterized organelle can induce NLRP3 inflammasome activation. In our previous study, we demonstrated that activation of the NLRP3 inflammasome mediates podocyte injury [6].

Silent mating type information regulation 2 homolog 1 (SIRT1) is an NAD+-dependent deacetylase that regulates many physiological effects. In mammals, SIRT1 is primarily localized in the nuclei, and most effects of SIRT1 depend on its deacetylation activity. In the kidney, SIRT1 is documented to have renoprotective functions and regulates glucose or lipid metabolism, such as diabetic nephropathy $[7,8]$. SIRT1 also has protective effects, such as maintenance of glomerular barrier function, regulation of mitochondrial function, energy metabolism, and antifibrosis effects. Some studies have reported the localization of SIRT1 in podocytes [7] and also explored the protective effect of SIRT1 on podocytes $[7,9$, 10]; however, the molecular mechanism of SIRT1's effects on aldosterone-induced podocyte injury remains unclear.

SIRT1 regulates longevity, possibly through maintenance of mitochondrial homeostasis. Mitochondrial health can be moderated in several ways, such as regulation and elimination of ROS through antioxidants and augmentation of mtDNA repair. In our previous study, SIRT1 was demonstrated to increase mitochondrial biogenesis and reduce ROS production through the transcription factor peroxisome proliferator-activated receptor-g coactivator $1 \alpha$ (PGC-1 $\alpha)$ [11].

In the present study, we found that the protective effect of SIRT1 on podocytes resulted from blocking the NLRP3 inflammasome by suppressing $\mathrm{MtD}$. These results suggest further targets for therapeutic interventions to treat glomerular diseases.

\section{Materials and Methods}

\section{Reagents and Antibodies}

Antibodies against SIRT1, caspase-1, and horseradish peroxidase-conjugated secondary antibodies were purchased from Santa Cruz Biotechnology (Santa Cruz, CA, USA). Anti- $\beta$-actin and fluorescently conjugated secondary antibodies were obtained from Cell Signaling Technology (Beverly, MA, USA). Antibodies against nephrin, podocin, and NLRP3 were purchased from Abcam (Cambridge, MA, USA). Collagenase A was purchased from Roche (Roche, Mannheim, Germany). Deoxyribonuclease I and Hanks' balanced salt solution (HBSS) were obtained from Invitrogen (Invitrogen, Lidingo, Sweden).

\section{Patients}

Renal biopsy samples were obtained from patients with CKD who were undergoing diagnostic evaluation at the Department of Nephrology, Children's Hospital of Nanjing Medical University. Renal biopsy samples were collected based on the criterion of having at least ten glomeruli in a paraffin-embedded tissue sample available for histological sectioning. A total of 18 subjects (age range: 1-14 years) were enrolled and pathologically diagnosed with membrane nephropathy (MN), minimal change disease (MCD), or focal segmental glomerulosclerosis (FSGS) (the details are presented in Table 1). Normal renal tissues were collected from the patients without proteinuria who received a partial nephrectomy for a benign renal tumor. The protocol for the use of biopsied samples and nephrectomized tissues from patients was approved by the local committee on human subjects at Children's Hospital of Nanjing Medical University (study approval number: 201703048). Written informed consent was provided by guardians of patients.

\section{Cell Culture and Plasmid Transfection}

MPC5 conditionally immortalized mouse podocyte clonal cells (provided by Peter Mundel of the Mount Sinai School of Medicine through Dr. Jie Ding at Peking University) were cultured and induced to differentiate as described previously [11]. The cells were maintained in Roswell Park Memorial Institute (RPMI) 1,640 medium (Hyclone, South Logan, UT, USA) containing 10\% heat-inactivated fetal calf serum (Gibco, Gaithersburg, MD, USA), $100 \mathrm{U} /$ $\mathrm{mL}$ penicillin $\mathrm{G}$, and $100 \mathrm{mg} / \mathrm{mL}$ streptomycin in a $5 \% \mathrm{CO}_{2}$ atmosphere. To sustain podocyte proliferation, $10 \mathrm{U} / \mathrm{mL}$ of recombinant murine interferon- $\gamma$ (Sigma, St. Louis, MO, USA) was added to the medium, and the cells were maintained at $33^{\circ} \mathrm{C}$. Podocytes were maintained without interferon $-\gamma$ at $37^{\circ} \mathrm{C}$ for $10-14$ days to induce differentiation before the experiments. In certain experiments, cells were transfected with SIRT1 plasmids for $24 \mathrm{~h}$ and then treated with aldosterone (200 nM).

\section{Primary Cultured Podocytes}

Isolation procedures were based on the report of Harris et al. [12]. The kidneys of wild-type (WT) and conditional knockout (cKO) of Sirt1 mice (20-30 days) were removed after sacrifice. Renal cortices were sliced into pieces of $\sim 1 \mathrm{~mm}$ width in an ice-cold dissection solution and digested in collagenase $(1 \mathrm{mg} / \mathrm{mL}$ collagenase $\mathrm{A}, 100 \mathrm{U} / \mathrm{mL}$ deoxyribonuclease I in $\mathrm{HBSS}$ ) at $37^{\circ} \mathrm{C}$ for $15 \mathrm{~min}$ with gentle agitation. The collagenase-digested tissue was gently pressed through a $100-\mu \mathrm{m}$ cell strainer using a flattened pestle, and the cell strainer was then washed with $5 \mathrm{~mL}$ of HBSS. The filtered cells were passed through a new cell strainer without pressing, and 
Table 1. The basic information and diagnosis of renal biopsy specimens

\begin{tabular}{llllll}
\hline Gender & $\begin{array}{l}\text { Age, } \\
\text { years }\end{array}$ & $\begin{array}{l}\text { Proteinuria, } \\
\text { g/24h }\end{array}$ & $\begin{array}{l}\text { Pathological } \\
\text { diagnosis }\end{array}$ & $\begin{array}{l}\text { Serum creatine, } \\
\mu \mathrm{mol} / \mathrm{L}\end{array}$ & $\begin{array}{l}\text { BUN, } \\
\mathrm{mmol} / \mathrm{L}\end{array}$ \\
\hline Boy & 4.2 & 1.62 & MCD & 19 & 2.86 \\
Boy & 4.8 & 1.43 & MCD & 21 & 3.22 \\
Boy & 2.2 & 1.59 & MCD & 38 & 2.42 \\
Boy & 3.9 & 0.14 & MCD & 26 & 5.01 \\
Boy & 4.9 & 2.21 & MCD & 33 & 2.45 \\
Boy & 4.2 & 0.02 & MCD & 28 & 409 \\
Girl & 1.7 & 3.29 & FSGS & 21 & 2.53 \\
Boy & 4.3 & 2.47 & FSGS & 33 & 5.99 \\
Girl & 2.0 & 3.23 & FSGS & 27 & 6.07 \\
Boy & 2.2 & 3.46 & FSGS & 21 & 7.24 \\
Boy & 10.5 & 4.60 & FSGS & 28 & 5.10 \\
Boy & 12.5 & 8.60 & FSGS & 27 & 24.32 \\
Girl & 4.5 & 0.81 & FSGS & 525 & 3.60 \\
Girl & 10.8 & 0.30 & MN & 38 & 2.92 \\
Girl & 13.0 & 0.90 & MN & 35 & 2.85 \\
Boy & 11.0 & 0.86 & MN & 41 & 5.60 \\
Girl & 6.0 & 5.76 & MN & 35 & 2.14 \\
Boy & 4.2 & 0.67 & MN & 26 & \\
\hline
\end{tabular}

MN, membrane nephropathy; MCD, minimal change disease; FSGS, focal segmental glomerulosclerosis.

the cell strainer was washed with $5 \mathrm{~mL}$ of HBSS. The cell suspension was centrifuged at $200 \mathrm{~g}$ for $5 \mathrm{~min}$. Isolated glomeruli were cultured on type I collagen-coated culture dishes in Dulbecco's modified Eagle's medium/F-12 (1:1) containing 5\% fetal bovine serum (Cansera International, Ontario, Canada) supplemented with $0.5 \%$ Insulin-Transferrin-Selenium-A liquid media supplement (Invitrogen), $100 \mathrm{U} / \mathrm{mL}$ penicillin, and $100 \mathrm{mg} / \mathrm{mL}$ streptomycin. Cultures were incubated at $37^{\circ} \mathrm{C}$ in a humidified incubator with $5 \% \mathrm{CO}_{2}$.

Subculture of primary cultured podocytes was performed after 3 days of culture of isolated glomeruli. Cellular outgrowths were detached with trypsin-ethylenediaminetetraacetic acid solution (Sigma) and passed through a $25-\mathrm{mm}$ sieve to remove the remaining glomerular cores. The filtered cells were cultured on collagen I-coated dishes or glass coverslips for one more day and processed for immunohistochemical analysis or RNA preparation. Primary cultured podocytes were $4 \%$ formaldehyde fixed $(10 \mathrm{~min}$ ) and then incubated in $1 \% \mathrm{BSA} / 10 \%$ normal goat serum $/ 0.3 \mathrm{M}$ glycine in $0.1 \%$ PBS-Tween for $1 \mathrm{~h}$. The cells were then incubated with the WT-1 antibody overnight at $4^{\circ} \mathrm{C}$ and then washed with PBS 3 times, followed by diluted secondary antibody at $37^{\circ} \mathrm{C}$ for $1 \mathrm{~h}$. The Coplin jar was covered with aluminum foil to protect the slides from the light. Fluorescent images were produced on a Zeiss Axioplan2 imaging microscope (Zeiss, Oberkochen, Germany).

Generation of the podocyte-specific deletion of Sirt1 knockout mice and genotyping mice with the floxed Sirt1 gene and Nphs2cre mice were purchased from the Jackson Laboratory (Bar Harbor, ME, USA) on a genetic background of C57BL/6 J. To generate the podocyte-specific deletion of Sirt1 knockout mice, homozygous mice with Sirt $1^{\text {floxflox }}$ were crossed with Nphs2-cre mice to get Sirt $1^{\text {flox/+ }}$ Nphs $2^{\text {cre(+) }}$ mice. Then, Sirt $1^{\text {flox/+ }} \mathrm{Nphs} 2^{\text {cre(+) }}$ mice were crossed with Sirt $1^{\text {floxflox }}$ mice to get Sirt $1^{\text {flox/flox }} \mathrm{Nphs} 2^{\text {cre }(+)}$ male
Table 2. The sequences of the primers used in the study

\begin{tabular}{ll}
\hline Primer name & Primer sequence $5^{\prime}-3^{\prime}$ \\
\hline SIRT1-F & CAGTGTCATGGTTCCTTTGC \\
SIRT1-R & CACCGAGGAACTACCTGAT \\
Nephrin-F & TTCAGACCACACCAACATCC \\
Nephrin-R & AGCCAGGTTTCCACTCCA \\
Podocin-F & GTGAGGAGGGCACGGAAG \\
Podocin-R & AGGGAGGCGAGGACAAGA \\
GAPDH-F & AATGGATTTGGACGCATTGGT \\
GAPDH-R & TTTGCACTGGTACGTGTTGAT \\
18S rRNA-F & TTCGGAACTGAGGCCATGATT \\
18S rRNA-R & TTTCGCTCTGGTCCGTCTTG \\
mtDNA-F & TTTTATCTGCATCTGAGTTTAATCCTGT \\
mtDNA-R & CCACTTCATCTTACCATTTATTATCGC \\
\end{tabular}

SIRT1, silent mating type information regulation 2 homolog 1 ; mtDNA, mitochondrial DNA.

mice (cKO). All male littermates that were negative for the Cre transgene served as WT controls. A routine PCR protocol was used for genotyping tail DNA with the following primer pairs: Sirt1 genotyping, forward: GGT TGA CTT AGG TCT TGT CTG and reverse: CGT CCC TTG TAA TGT TTC CC, which yielded 550- and 750-bp bands, respectively, for the floxed and WT alleles; Cre transgene genotyping, forward: GCG GTC TGG CAG TAA AAA CTA TC and reverse: GTG AAA CAG CAT TGC TGT CAC TT, which generated a 100-bp fragment. 


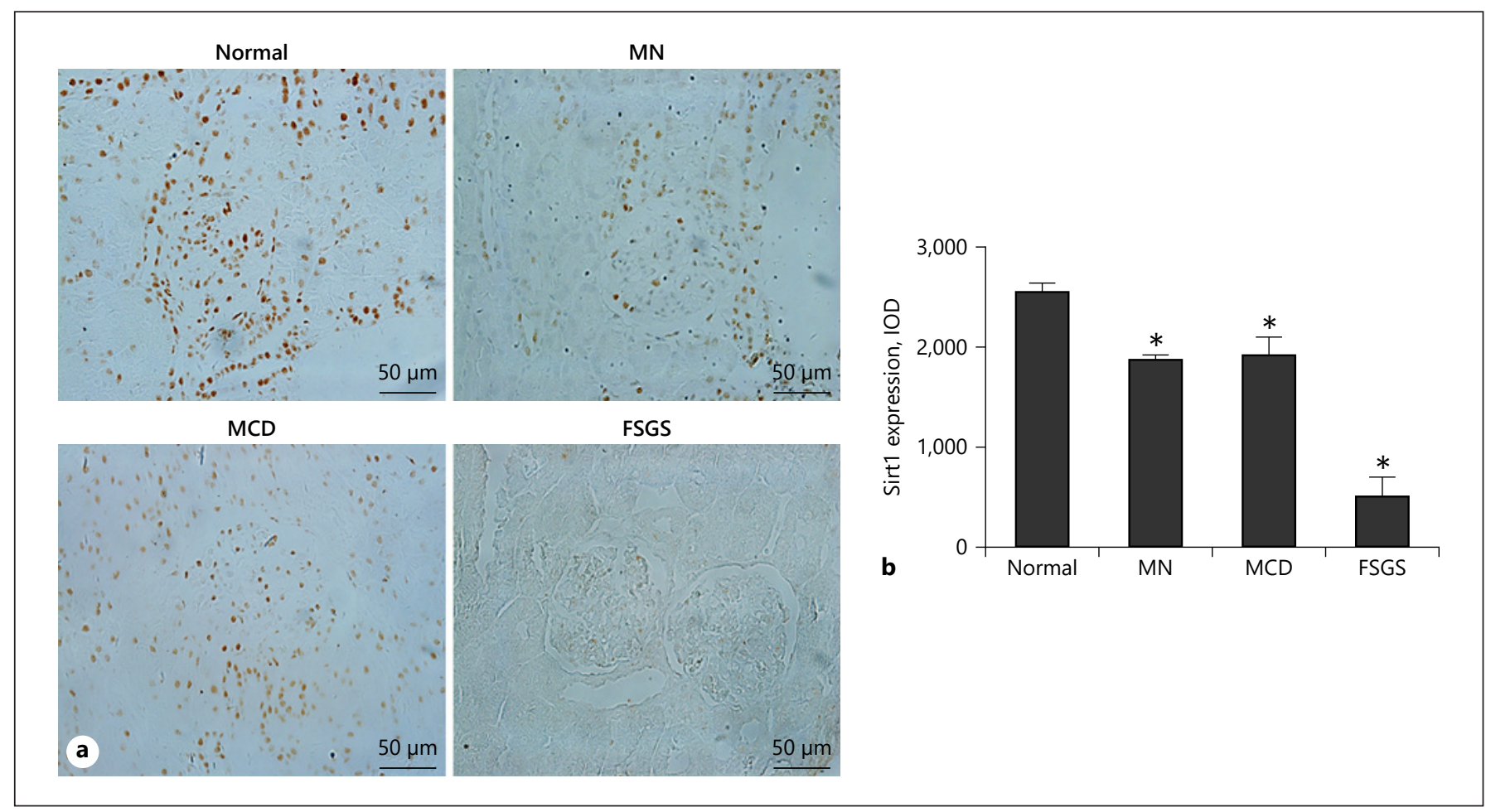

Fig. 1. Expression of SIRT1 in human kidney tissues. a Representative images of SIRT1 expression by immunohistochemistry in renal biopsy specimen diagnosed with MN, MCD, and FSGS. b IOD analysis of SIRT1 immunohistochemistry. Results are presented as means \pm SEM $(n=6) .{ }^{*} p<0.05$ versus normal. SIRT1, silent mating type information regulation 2 homolog 1; MN, membrane nephropathy; MCD, minimal change disease; FSGS, focal segmental glomerulosclerosis.

\section{Animal Experiments}

The animal study protocols were reviewed and approved by the Institutional Animal Care and Use Committee at Nanjing Medical University, China. In brief, 8-12-week-old WT and cKO male mice were chosen, weighing 25-30 g. Mice were treated with aldosterone using osmotic subcutaneously implanted minipumps. The pumps were placed by making an incision in the right flank region under light 3\% isoflurane anesthesia. The pumps delivered a continuous infusion of aldosterone $(300 \mu \mathrm{g} / \mathrm{kg} /$ day) (Alzet, Durect, Cupertino, CA, USA) for 14 days. All mice were maintained on a 12 -h light-dark cycle in a temperature-controlled $\left(19-21^{\circ} \mathrm{C}\right)$ room and were fed standard rodent chow.

\section{Quantitative Real-Time PCR}

Total RNA from cultured podocytes and isolated glomeruli were extracted using the TRIzol reagent (Invitrogen). Oligonucleotides were designed using Primer3 software (available at http:// frodo.wi.mit.edu/) and synthesized by Invitrogen. PCR was used to detect the mtDNA copy number and the target gene expression. Reverse transcription was performed using a Transcriptor First Stand cDNA Synthesis kit (Roche, Mannheim, Germany) according to the manufacturer's protocol. Real-time PCR amplification was performed using an ABI 7500 Real-time PCR Detection System (Applied Biosystems, Foster City, CA, USA) with a FastStart Universal SYBR Green master mix (Roche). The cycling condi- tions were $95^{\circ} \mathrm{C}$ for $10 \mathrm{~min}$, followed by 40 cycles of $95^{\circ} \mathrm{C}$ for $15 \mathrm{~s}$ and $60^{\circ} \mathrm{C}$ for $1 \mathrm{~min}$. The relative $\mathrm{mtDNA}$ copy numbers were normalized to the $18 \mathrm{~S}$ ribosomal RNA levels encoded by the nuclear DNA, and cycle threshold values were used to analyze the mRNA levels using SDS 2.2.2 software (Applied Biosystems). The primers are shown in Table 2.

\section{Western Blotting}

Podocytes or isolated glomeruli were lysed in protein lysis buffer, and the protein concentration was measured as previously reported [6]. Immunoblotting was performed using primary antibodies against nephrin $(1: 1,000)$, podocin $(1: 1,000)$, SIRT1 (1:500), NLRP3 (1:1,000), and caspase-1 (1:1,000). The blots were visualized using the Amersham ECL Detection System (Amersham, Buckinghamshire, UK), and a densitometric analysis was performed using Quantity One Software (Bio-Rad, Hercules, CA, USA).

\section{Analysis of Urinary Albumin}

The urinary concentration of albumin was determined using ELISA kits from Exocell (Philadelphia, PA, USA) and corrected by urine Cr.

\section{Immunoreactivity and Histological Analysis}

For the histological analysis, harvested kidneys from mice were fixed overnight in $4 \%$ paraformaldehyde at $4^{\circ} \mathrm{C}$, embedded in par- 


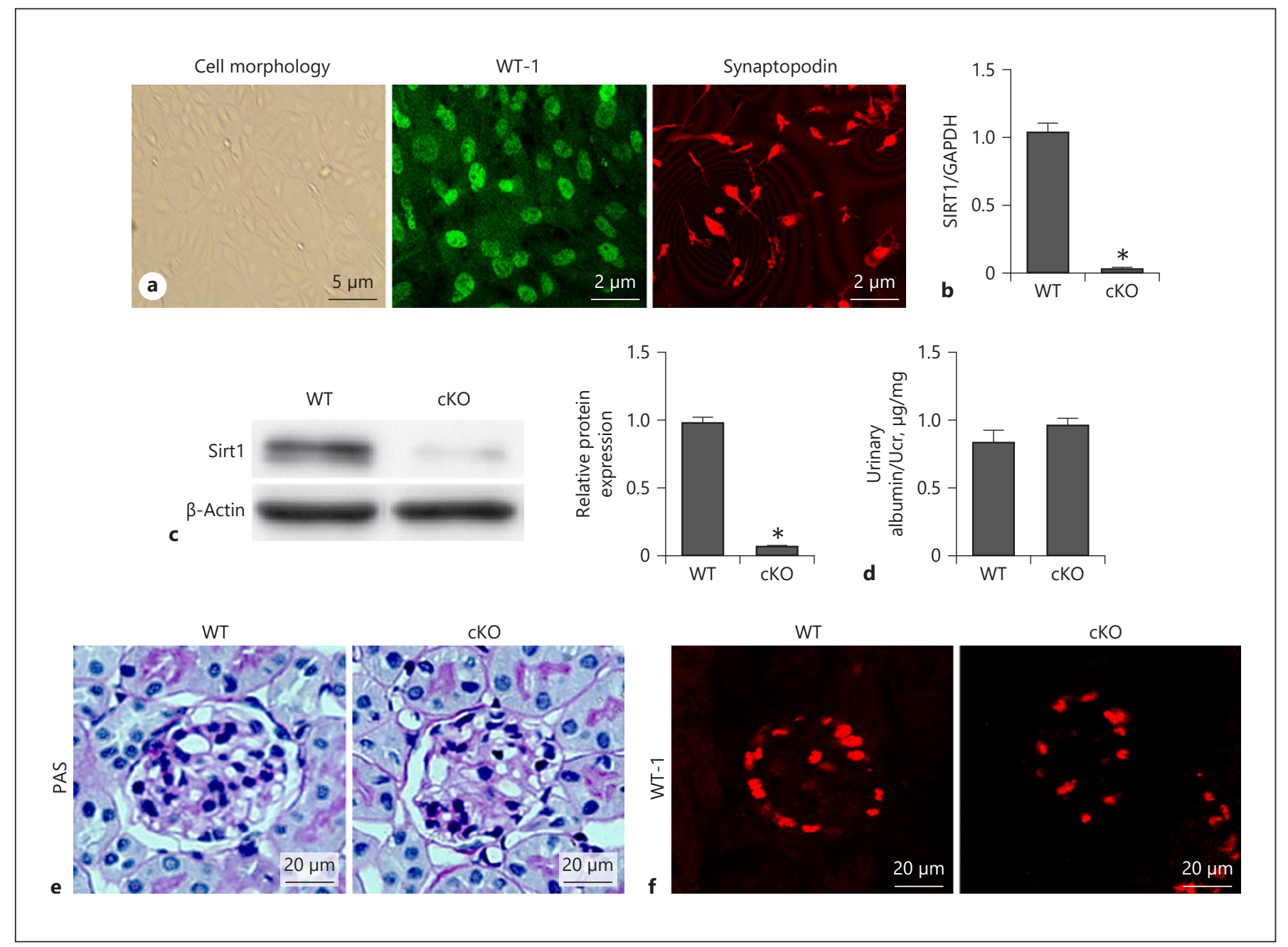

Fig. 2. Specific podocyte Sirt1 conditional knockout mice were generated. Identification of podocyte primary culture cell morphology (a, left) and WT-1 (a, middle) and synaptopodin (a, right) expression by immunofluorescence. Sirt 1 mRNA level by qRTPCR (b). SIRT1 protein levels in podocyte primary culture cells from $\mathrm{cKO}$ mice and WT controls determined by Western blot (c).
Measurement of urine albumin levels (albumin-to-Cr ratio) (d), PAS staining (e), and WT-1 expression between WT and cKO mice $(\mathbf{f})$. Results are presented as means $\pm \operatorname{SEM}(n=6) .{ }^{*} p<0.05$ versus the WT group. SIRT1, silent mating type information regulation 2 homolog 1; WT, wild type; cKO, conditional knockout; PAS, periodic acid-Schiff. affin, and sectioned transversely. The kidneys used for the immunofluorescence analysis were embedded in Tissue-Tek ${ }^{\circledR}$ O.C.T. compound (Sakura Inetek Europe BV, Alphen aan den Rijn, Netherlands). Cryosections were cut to a thickness of $8 \mu \mathrm{m}$, and immunostaining was carried out as described previously [6]. Fluorescent images were produced on a Zeiss Axioplan2 imaging microscope (Zeiss, Oberkochen, Germany). Kidney sections $(3 \mu \mathrm{m})$ were stained with periodic acid-Schiff.

\section{Immunohistochemical Analysis}

Kidneys were fixed in $4 \%$ paraformaldehyde and embedded in paraffin. Paraffin sections of each specimen were cut at a thickness of $3 \mu \mathrm{m}$, followed by a standard protocol, using xylene and graded ethanol to deparaffinize and rehydrate the tissue. These sections were washed with PBS and treated with blocking buffer containing $50 \mathrm{mM} \mathrm{NH}_{4} \mathrm{Cl}, 2 \% \mathrm{BSA}$, and $0.05 \%$ saponin in PBS for $20 \mathrm{~min}$ at room temperature. The sections were then incubated overnight at $4^{\circ} \mathrm{C}$ with anti-SIRT1 antibodies (1:100). After washing with PBS, the secondary antibody was applied, and the signals were visualized using an ABC kit (Santa Cruz Biotechnology).

\section{Transmission Electron Microscopy}

Kidney cortexes were cut into $1 \times 1 \times 1 \mathrm{~mm}$ pieces and then postfixed in $5 \%$ glutaraldehyde. Ultrathin sections $(60 \mathrm{~nm})$ were cut on a microtome, placed on copper grids, stained with uranyl acetate and lead citrate, and examined under an electron microscope (JEOL JEM-1010, Tokyo, Japan). 

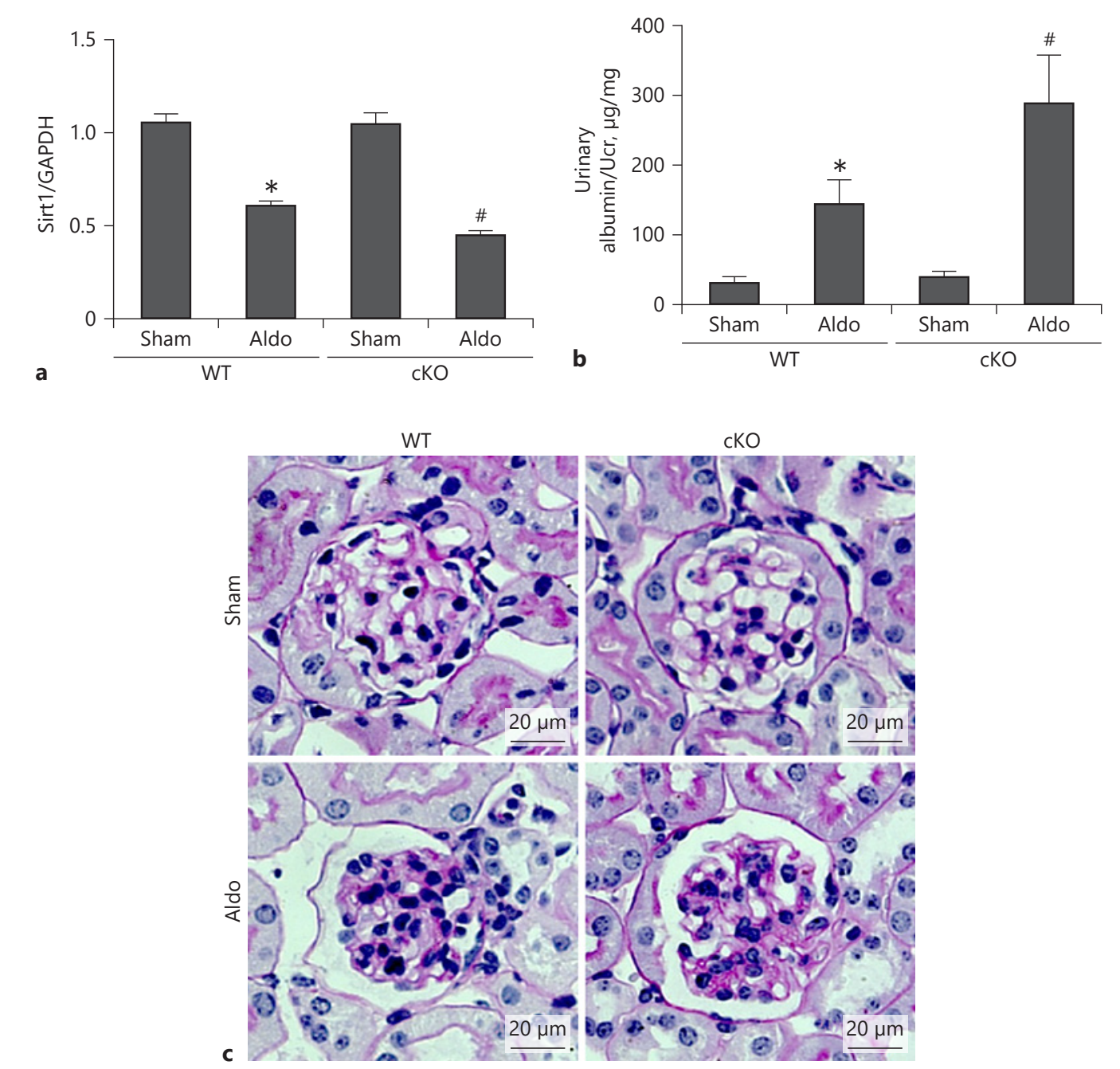

Fig. 3. Podocyte conditional Sirt1 knockout aggravated Aldo-induced albuminuria and renal pathology. Male WT and cKO mice were infused with Aldo $(300 \mu \mathrm{g} / \mathrm{kg} /$ day $)$ for 2 weeks. a qRT-PCR analysis of Sirt1. Measurement of urine albumin levels (albumin-to-Cr ratio) (b) and PAS staining (c). Results are presented as means \pm $\operatorname{SEM}(n=6) .{ }^{*} p<0.05$ versus the WT + sham group. $\# p<0.05$ versus WT + Aldo. Sirt1, silent mating type information regulation 2 homolog 1; WT, wild type; cKO, conditional knockout; PAS, periodic acid-Schiff.

Statistical Analysis

All results are presented as the means \pm the standard error of the mean. Data were compared between 2 groups using a 1-way ANOVA, whereas multiple groups were compared using the Kruskal-Wallis test performed with SPSS 13.0 statistical software (SPSS, Armonk, NY, USA). A value of $p<0.05$ indicated statistical significance.

\section{Results}

\section{Reduced SIRT1 Expression in Primary}

Podocytopathies

SIRT1 is reported to have protective functions on kidney diseases; however, the molecular mechanism of the function of SIRT1 in podocytes remains unclear. To explore the relationship between SIRT1 expression and human disease progression, we examined SIRT1 expression 


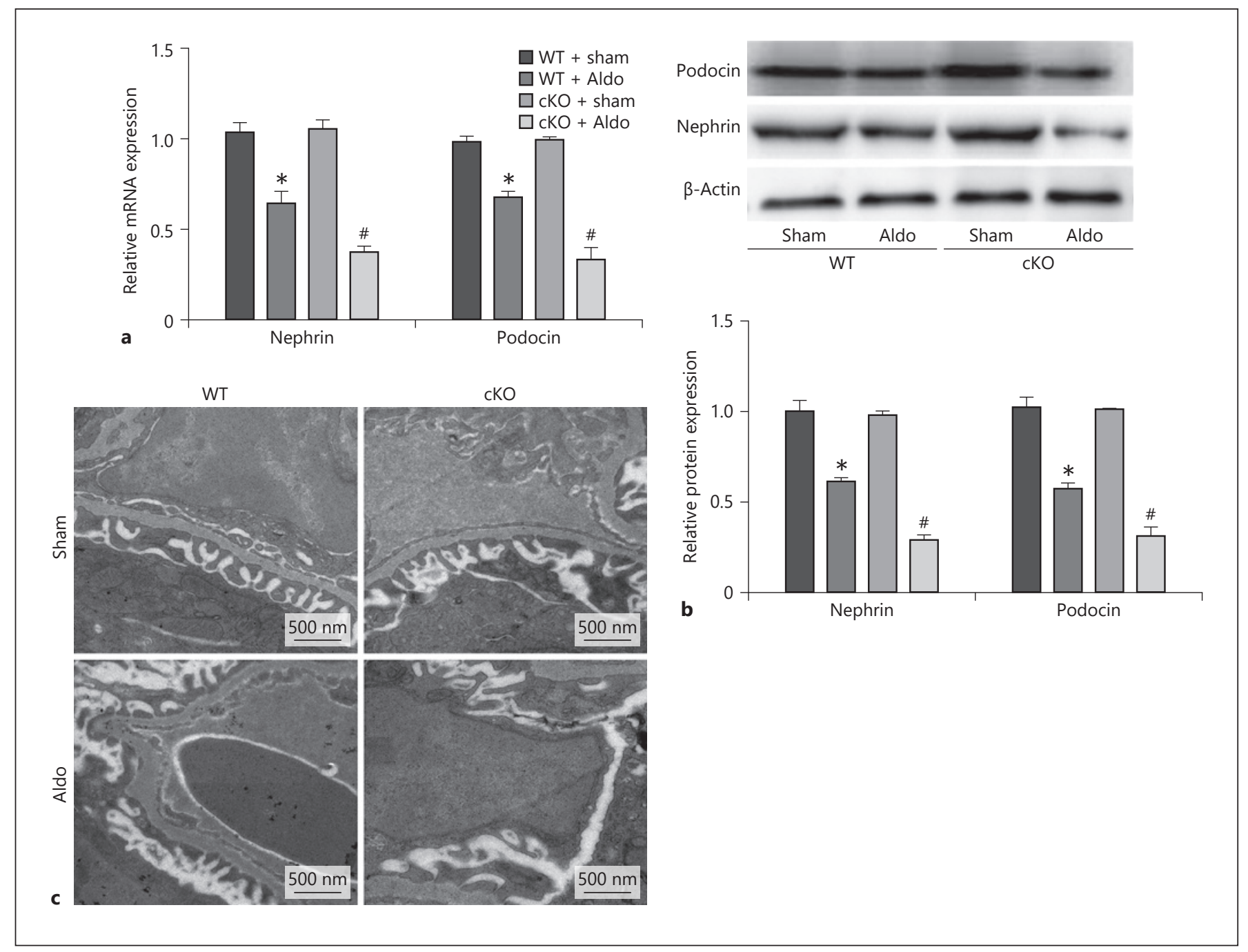

Fig. 4. Podocyte conditional Sirt1 knockout aggravated Aldo-induced podocyte injury. qRT-PCR analysis of nephrin and podocin in the kidney cortex (a) and Western blotting analysis for nephrin and podocin (b). Upper: representative immunoblots. Lower: densitometric analysis. Electron microscopy of podocyte foot (c). Results are presented as means $\pm \operatorname{SEM}(n=6) .{ }^{*} p<0.05$ versus the $\mathrm{WT}+$ sham group. $\# p<0.05$ versus WT + Aldo. Sirt 1 , silent mating type information regulation 2 homolog 1; WT, wild type.

in renal biopsy specimens from patients with primary podocytopathies, including FSGS, MCD, and MN by immunohistochemistry. As shown in Figure 1a, SIRT1 levels gradually decreased with the increased severity of podocyte pathological damage. In the MN and MCD groups, SIRT1 was reduced by $30 \%$ compared with the normal group. In the FSGS group, SIRT1 levels were about $20 \%$ of that in the Normal group (Fig. 1b). This result suggested that SIRT1 might play an important role in podocyte diseases.

SIRT1 Inhibits NLRP3 Inflammasome Activation

\section{Generation of Specific Podocyte Sirt1 cKO Mice}

In this study, specific podocyte Sirt 1 cKO mice were generated. We used Sirt $1^{\text {flox/flox }}$ mice crossed with podocin-Cre mice. We extracted the primary podocytes and used WT-1 and synaptopodin staining to verify their purity (Fig. 2a). As shown in Figure 2b and c, Sirt1 knockout in podocytes was confirmed by Western blotting and real-time PCR. Sirt 1 cKO mice did not show any physiological (including albuminuria and podocyte number) or histological changes compared with WT mice (Fig. 2d-f). 


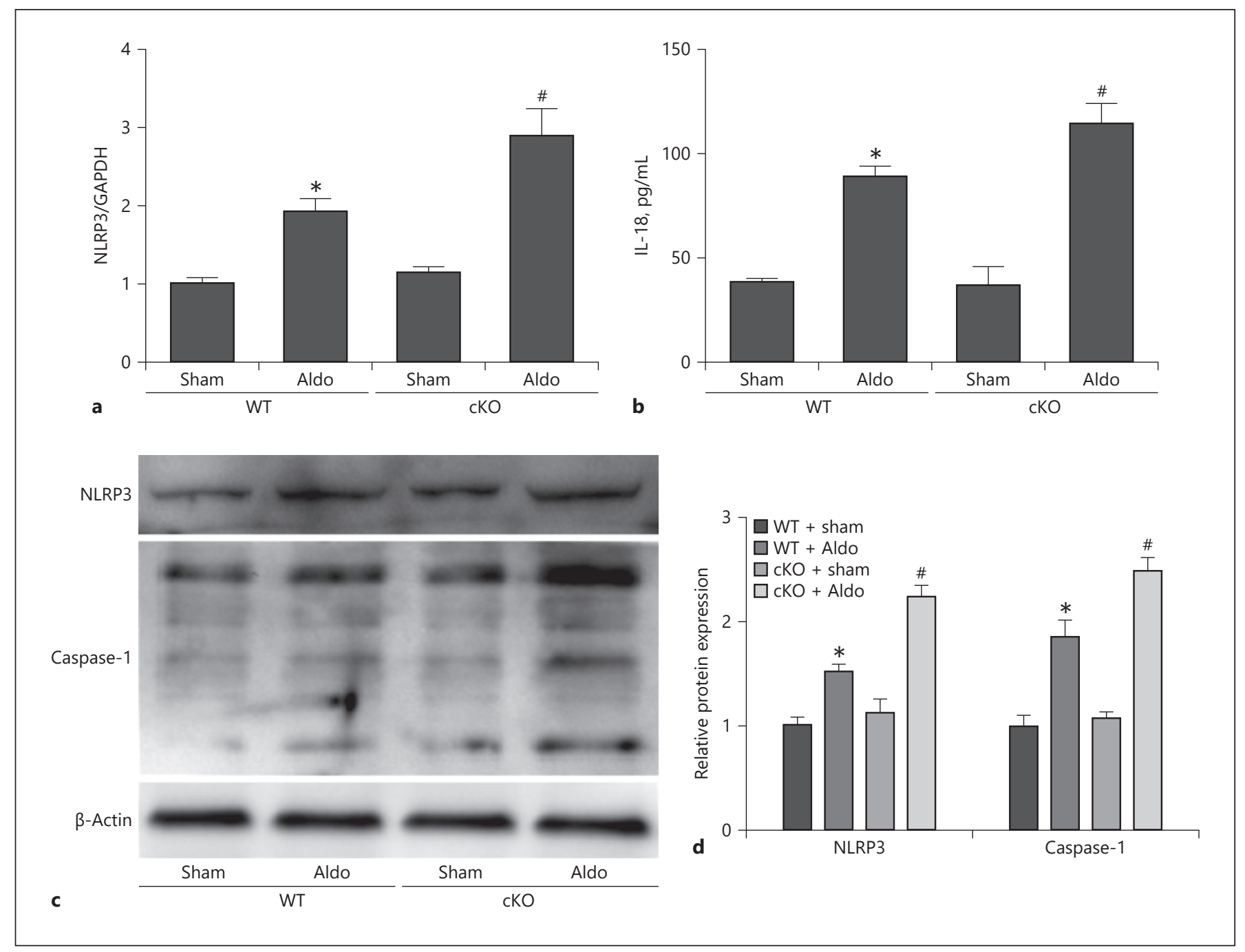

Fig. 5. Loss of Sirt1 in podocyte activated NLRP3 inflammation. Mice were treated with a continuous infusion of Aldo for 14 days. NLRP3 mRNA level by qRT-PCR analysis (a), ELISA analysis of urine IL-18 levels (b), and representative immunoblots of Western blotting analysis for NLRP3 and caspase-1 (c) and densitometric analysis $(\mathbf{d})$. Results are presented as means $\pm \operatorname{SEM}(n=6) .{ }^{*} p<0.05$ versus the $\mathrm{WT}+$ sham group. $\# p<0.05$ versus WT + Aldo. Sirt1, silent mating type information regulation 2 homolog 1; WT, wild type; NLRP3, NLR family pyrin domain containing 3 .

\section{Podocyte Conditional SIRT1 Knockout Exacerbated} Aldo-Induced Albuminuria and Renal Pathology

In this study, we used an aldosterone-infused model to induce podocyte injury. Aldosterone was used at $300 \mu \mathrm{g} /$ $\mathrm{kg}$ /day for 14 days by minipumps. We detected the expression of Sirt 1 in 4 groups. Since total RNA was extracted from the renal cortex and SIRT1 in other types of renal cells except for podocytes was not deleted, Sirt1 expression in cortex tissue was not significantly decreased in the cKO Sham group, but it was slightly lower than that in the WT mice after treatment by Aldo (Fig. 3a). As shown in
Figure $3 \mathrm{~b}$, after aldosterone infusion, urinary/uCr was significantly higher in cKO mice than WT mice. Periodic acid-Schiff staining showed more serious glomerular enlargement and increased mesangial area in $\mathrm{cKO}$ mice compared with that in the WT mice (Fig. 3c).

\section{Podocyte Conditional Sirt1 Knockout Aggravated Aldosterone-Induced Podocyte Injury}

Next, we further examined the expression of nephrin and podocin. Real-time PCR and Western blotting showed that aldosterone significantly decreased nephrin 

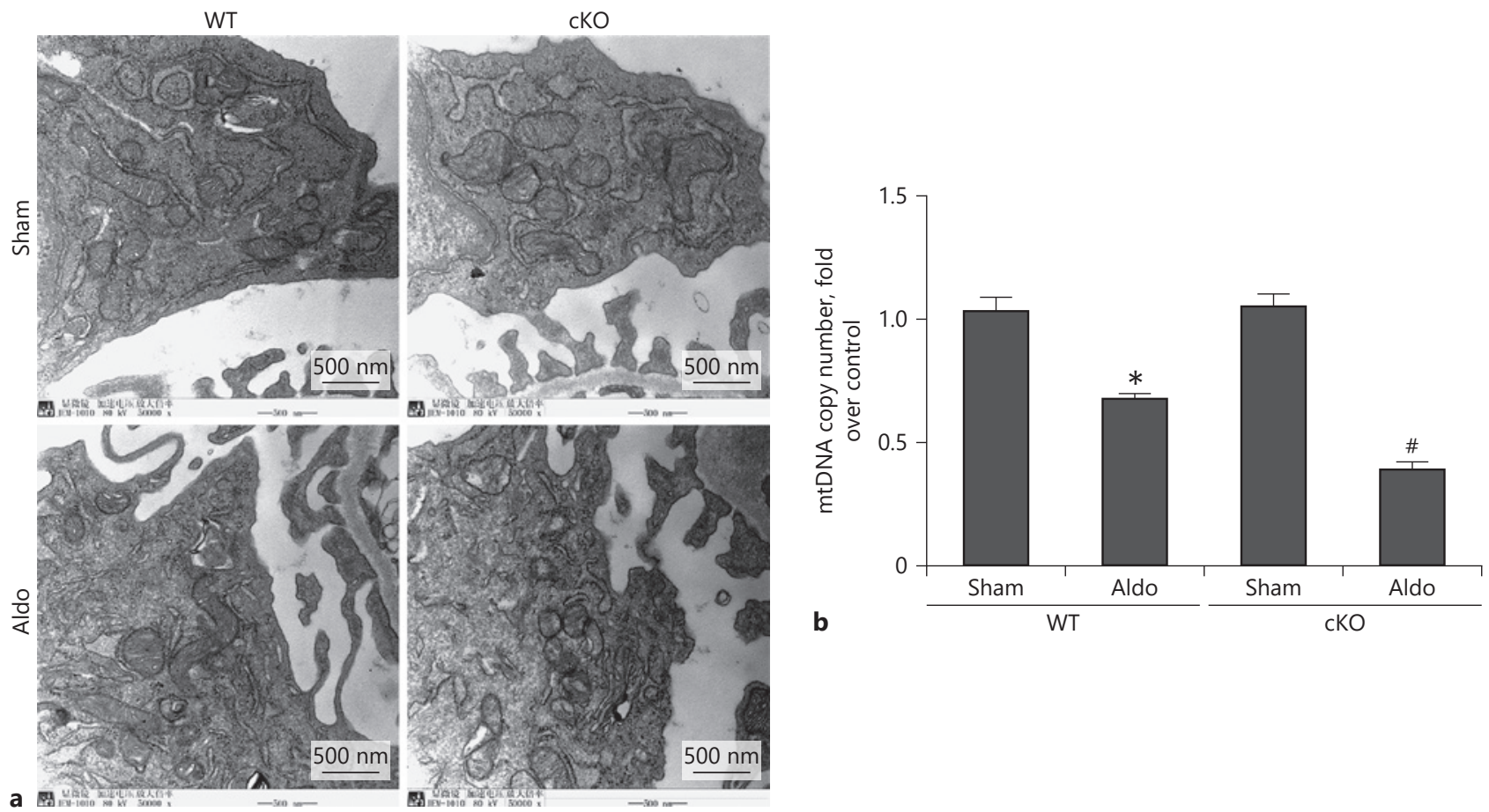

Fig. 6. Deletion of Sirt1 in podocyte-aggravated MtD. Mitochondrial morphology in glomerular podocytes $(\times 50,000)(\mathbf{a})$ and mtDNA copy number $(\mathbf{b})$. Results are presented as means \pm SEM $(n=6) .{ }^{*} p<0.05$ versus the $\mathrm{WT}+$ sham group. $\# p<0.05$ versus WT + Aldo. Sirt1, silent mating type information regulation 2 homolog 1; WT, wild type; mtDNA, mitochondrial DNA; MtD, mitochondrial dysfunction.

and podocin levels in WT mice and remarkably aggravated podocyte injury in the $\mathrm{cKO}$ mice compared with the WT mice (Fig. 4a, b). In electron micrographs, the fusion of foot processes was evident and the width of podocyte filtration slits was decreased in Aldo-treated WT mice. In the cKO mice, fusion of foot processes was more extensive and the width of podocyte filtration slits was further reduced after aldosterone infusion compared with the WT mice (Fig. 4c). These results provided more solid evidence indicating that deletion of Sirt1 in podocytes could deteriorate podocyte injury induced by aldosterone.

\section{Loss of Sirt1 in Podocyte Activated NLRP3 Inflammation}

To determine whether deletion of Sirtl aggravated aldosterone-induced podocyte injury through NLRP3 inflammasome activation, we determined the NLRP3, interleukin (IL)-18, and caspase-1 expression after Sirt1 knockout. We observed that aldosterone could induce activation of the NLRP3/caspase-1/IL-18 pathway. This effect was remarkably exacerbated in the Sirt1 knockout mice (Fig. 5a-d).

\section{Deletion of Sirt1 in Podocyte Aggravated MtD}

Our previous studies found that aldosterone could induce $\mathrm{MtD}$. Thus, we also evaluated the mitochondria status in aldosterone-infused kidneys. The results showed that mitochondria in the normal group were rod shaped, full, and regular in shape, with clear and orderly mitochondrial cristae. After aldosterone infusion in WT mice, the mitochondria in podocytes swelled and the cristae blurred or even disappeared. As expected, we found that deletion of Sirt1 in podocytes had enhanced swollen mitochondria with disorganized and fragmented cristae and further reduced mtDNA copy number (Fig. 6a, b). 


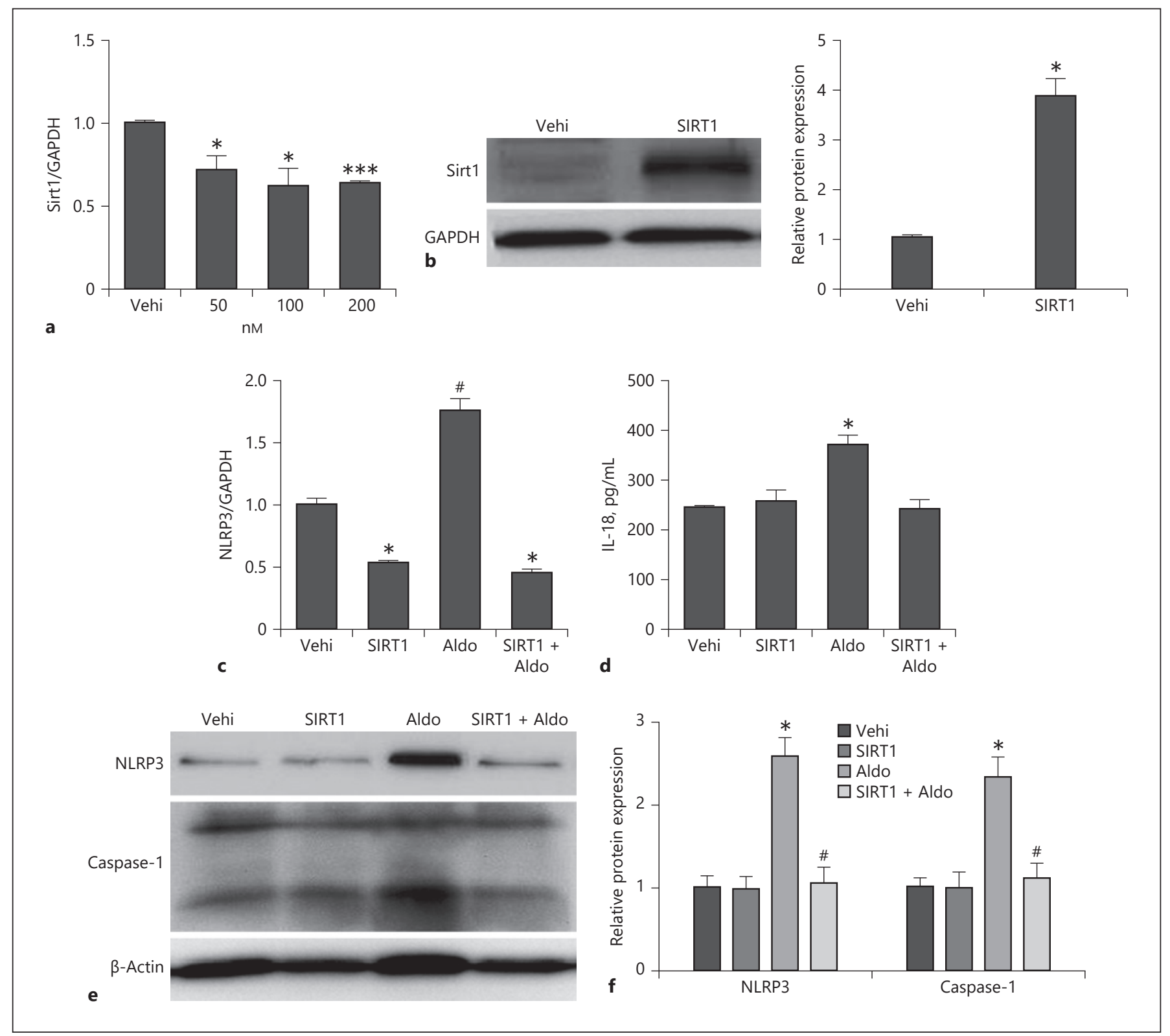

Fig. 7. SIRT1 overexpression inhibited NLRP3 inflammation activation in podocyte. Cells were grown on 6-well plates until $60-70 \%$ confluence, and cultured podocytes were treated with different concentrations of aldosterone (50, 100, and $200 \mathrm{nM})$. qRT-PCR analysis of Sirt1 (a). Cells were grown on 6-well plates until 50$60 \%$ confluence, transfected with Sirt1 or vehi plasmid for $24 \mathrm{~h}$, and then, treated with Aldo (200 nmol/L) for another $24 \mathrm{~h}$. Representative immunoblots of Western blotting analysis of overex- pressed SIRT1 and densitometric analysis (b), NLRP3 mRNA level (c), ELISA analysis of IL-18 levels in the medium (d), representative immunoblots of NLRP3 and caspase-1 protein levels (e), and densitometric analysis (f). Results are presented as means \pm SEM $(n=4) .{ }^{*} p<0.05$ versus the vehi group. $\# p<0.05$ versus the Aldo group. SIRT1, silent mating type information regulation 2 homo$\log 1$; NLRP3, NLR family pyrin domain containing 3; IL-18, interleukin-18.

\section{SIRT1 Overexpression Inhibited NLRP3 Inflammation} Activation

To detect the direct effect of SIRT1 in podocyte, we treated cultured podocytes with aldosterone $(50,100,200$
$\mathrm{nM}$ ) and found that Sirt1 expression decreased after Aldo stimulation (Fig. 7a). Then, Sirt1 was overexpressed to MPC5 stimulated by aldosterone. As shown by the data from quantitative RT-PCR, Western blots, and ELISA, we 


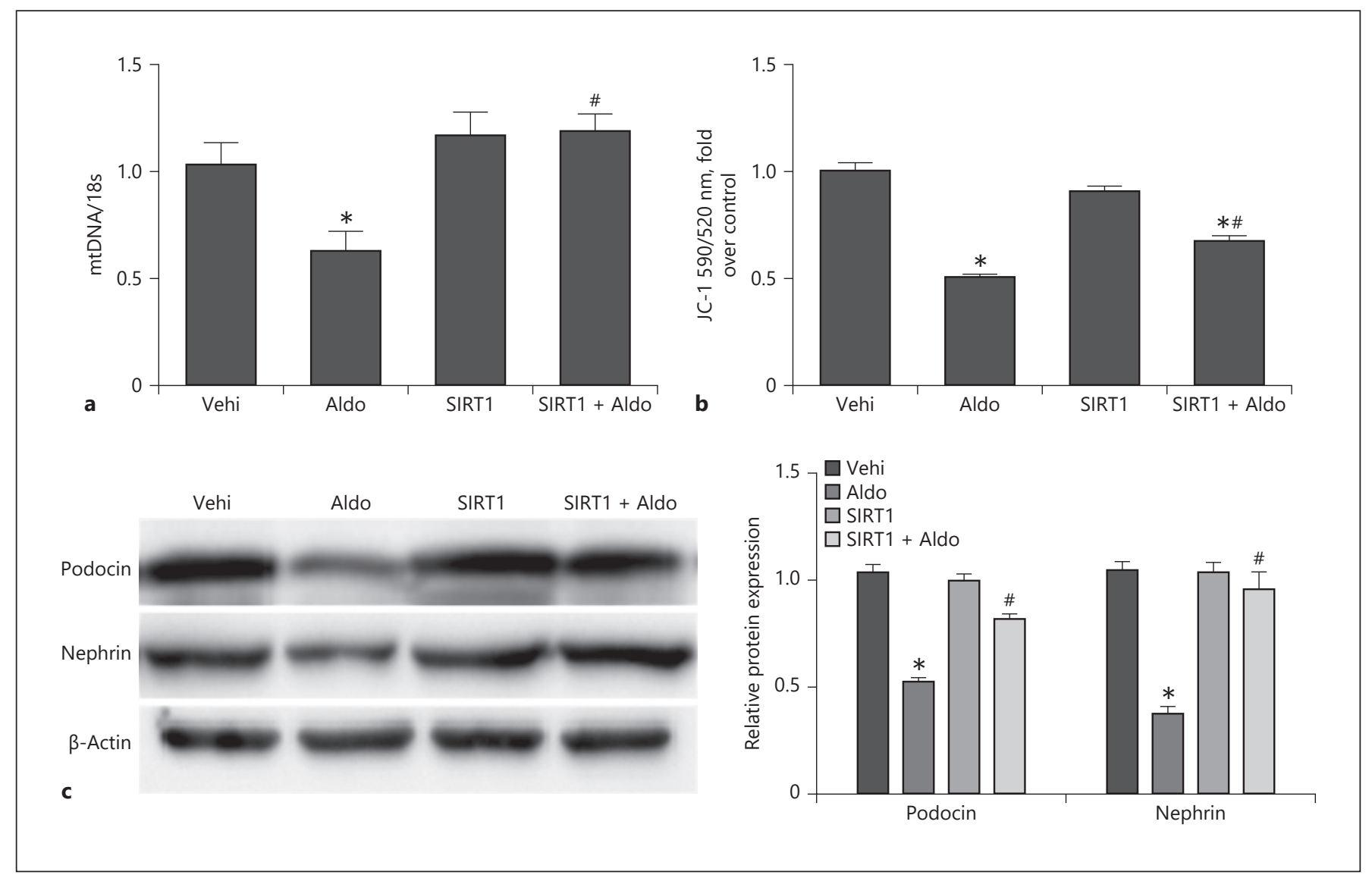

Fig. 8. Sirt1 overexpression inhibited MtD and podocyte injury. Cells were grown on 6-well plates until 50-60\% confluence, transfected with Sirtl or vehi plasmid for $24 \mathrm{~h}$, and then, treated with Aldo (200 nmol/L) for another 24 h. mtDNA copy number (a) and quantitation of JC-1 fluorescence by flow cytometry (b). Western blotting analysis of podocin and nephrin and densitometric analysis (c). Results are presented as means \pm SEM $(n=4)$. ${ }^{*} p<0.05$ versus the vehi group. $\# p<0.01$ versus the Aldo group. Sirt1, silent mating type information regulation 2 homolog 1 ; mtDNA, mitochondrial DNA; MtD, mitochondrial dysfunction.

found that aldosterone-induced NLRP3 inflammation activation and SIRT1 reversed this activation, including decreased expression of IL-18, caspase-1, and NLRP3 (Fig. $7 b-f$ ). These results indicated that SIRT1 inhibits NLRP3 inflammation activation.

\section{SIRT1 Overexpression Inhibited MtD and Podocyte Injury}

We also detected MtD markers, mtDNA and JC-1, and podocyte injury markers. We found that SIRT1 overexpression could reverse mtDNA and JC-1 decreases and then protect the podocytes from aldosterone-induced nephrin and podocin reduction (Fig. 8a-c). These data indicated that SIRT1 prevents $\mathrm{MtD}$, thereby protecting podocytes from aldosterone-induced injury.

SIRT1 Inhibits NLRP3 Inflammasome Activation

\section{Discussion}

In the present study, specific podocyte Sirt1 cKO mice were generated. The $\mathrm{cKO}$ mice exhibited no significant abnormalities in gene expression or pathology in the kidney. However, when we induced a podocyte injury model by aldosterone infusion, the $\mathrm{cKO}$ mice showed more serious glomerular enlargement and increased mesangial area. Aldosterone remarkably aggravated podocyte injury in the cKO mice compared with that in the WT mice. Specific knockout of Sirt1 in podocytes resulted in activation of NLRP3 inflammation. Overexpression of SIRT1 blocked NLRP3 activation and protected against MtD in podocytes. Therefore, our results revealed that SIRT1 protects against podocyte injury via suppressing $\mathrm{MtD}$ and preventing NLRP3 activation. 
A number of studies have reported that SIRT1 plays a protective role in injured podocytes $[7,13]$. In our previous study, we found that SIRT1 could regulate mitochondrial function through the SIRT1-PGC1 $\alpha$-mitochondrial axis [11]. Wen et al. [14] found that resveratrol attenuates diabetic nephropathy via modulating angiogenesis. Kume et al. [15] showed that SIRT1 expression was reduced in patients with diabetic nephropathy. Di Sante et al. [16] also demonstrated that lower SIRT1 expression in the luminal epithelium was associated with poor prognosis in human prostate cancer. In the current study, we found that SIRT1 expression was decreased in podocyte diseases such as FSGS, MCD, and MN, especially in FSGS. To detect the effect of SIRT1 on podocytes, we generated podocyte-specific Sirt1 knockout mice. Hasegawa demonstrated that knockout of Sirt1 in proximal tubular cells decreased the expression of SIRT1 in glomerular podocytes and increased the expression of a tight junction protein, claudin-1, which resulted in albuminuria [17]. We found that podocyte conditional Sirt1 knockout in mice resulted in aggravated podocyte injury after aldosterone infusion. Consistent with this result, Motonoishi found that podocyte Sirt1 knockout mice showed marked exacerbation of podocyte injury, including actin cytoskeleton derangement compared with WT mice [9].

The NLRP3 inflammasome is activated by a variety of danger signals, which mediate inflammation as an innate component. Evidence shows that NLRP3 inflammasome activation may regulate the development and process of glomerular sclerosis $[18,19]$. NLRP3 inflammasome activation may turn on glomerular inflammation and cause other cell damage, contributing to the onset of glomerular injury and ESRD. This inflammasome activation not only occurs in immune cells but also in residential cells, such as endothelial cells and podocytes in the glomeruli [20]. Li et al. [21] found that SIRT1 expression was reduced in human umbilical vein epithelial cells stimulated with lipopolysaccharide, and the lack of SIRT1-enhanced NLRP3 inflammasome activation and subsequent caspase- 1 cleavage. Li et al. [22] found that an SIRT1 activator decreased CD40/CD40L levels and serum IL-1 $\beta$ level in collared arteries. Ma et al. [23] showed that overexpression of SIRT1 suppressed nuclear factor kappa B/NLRP3 inflammasome activation in high fructose-fed rats. Although some studies have shown that SIRT1 could inhibit NLRP3, the role of SIRT1/NLRP3 in aldosteroneinduced podocyte injury has not been reported. In the present study, knockout Sirt1 in podocytes aggravated NLRP3 inflammasome activation. In cultured podocytes, overexpression of SIRT1 inhibited NLRP3 activation.
These findings showed that SIRT1 could attenuate the activation of the NLRP3 inflammasome and protect podocytes from aldosterone-induced injury.

In conclusion, we found that SIRT1 could inhibit NLRP3 inflammation activation through preventing $\mathrm{MtD}$ to protect against podocyte injury. Our results provide the first evidence showing the strong effect of SIRT1 on NLRP3 activation in aldosterone-induced podocyte injury, suggesting that the SIRT1/NLRP3 axis might be a potential target in the prevention and treatment of podocyte injury.

\section{Statement of Ethics}

The protocol for the use of biopsied samples and nephrectomized tissues from patients was approved by the local committee on human subjects at Children's Hospital of Nanjing Medical University. Written informed consent was provided by guardians of patients (study approval number: 201703048). All animal experiments were performed in accordance with the guidelines and regulations of the Animal Experimentation Ethics Committee of Nanjing Medical University. All experimental protocols were approved by the same committee.

\section{Conflict of Interest Statement}

There are no conflicts of interest.

\section{Funding Sources}

This work was supported by grants from the National Natural Science Foundation of China (Nos. 81970581, 81830020, 91742205, 81625004, and 81530023) and the National Key Research and Development Program (No. 2016YFC0906103).

\section{Author Contributions}

Jiang M. and Zhao M. performed the experiments and wrote the manuscript. Lei J., Yuan Y., Huang S., Zhang Y., Ding G., and Jia Z. helped design the experiments and interpreted the data. Bai M. revised the manuscript. Zhang A. designed, supervised, and revised the manuscript. 


\section{References}

1 Ichikawa I, Ma J, Motojima M, Matsusaka T. Podocyte damage damages podocytes: autonomous vicious cycle that drives local spread of glomerular sclerosis. Curr Opin Nephrol Hypertens. 2005 May;14(3):205-10.

2 Picard M, Taivassalo T, Gouspillou G, Hepple RT. Mitochondria: isolation, structure and function. J Physiol. 2011 Sep 15;589(Pt 18): 4413-21.

3 Pieczenik SR, Neustadt J. Mitochondrial dysfunction and molecular pathways of disease. Exp Mol Pathol. 2007 Aug;83(1):84-92.

4 Zhu C, Huang S, Yuan Y, Ding G, Chen R, Liu $B$, et al. Mitochondrial dysfunction mediates aldosterone-induced podocyte damage: a therapeutic target of PPARgamma. Am J Pathol. 2011 May;178(5):2020-31.

5 Su M, Dhoopun AR, Yuan Y, Huang S, Zhu C, Ding G, et al. Mitochondrial dysfunction is an early event in aldosterone-induced podocyte injury. Am J Physiol Renal Physiol. 2013 Aug 15;305(4):F520-31.

6 Bai M, Chen Y, Zhao M, Zhang Y, He JC, Huang S, et al. NLRP3 inflammasome activation contributes to aldosterone-induced podocyte injury. Am J Physiol Renal Physiol. 2017 Apr 1;312(4):F556-F64.

7 Hong Q, Zhang L, Das B, Li Z, Liu B, Cai G, et al. Increased podocyte Sirtuin-1 function attenuates diabetic kidney injury. Kidney Int. 2018 Jun;93(6):1330-43.

8 Wang W, Sun W, Cheng Y, Xu Z, Cai L. Role of sirtuin-1 in diabetic nephropathy. J Mol Med. 2019 Mar;97(3):291-309.

9 Motonishi S, Nangaku M, Wada T, Ishimoto Y, Ohse T, Matsusaka T, et al. Sirtuin1 maintains actin cytoskeleton by deacetylation of cortactin in injured podocytes. J Am Soc Nephrol. 2015 Aug;26(8):1939-59.
10 Zhang T, Chi Y, Ren Y, Du C, Shi Y, Li Y. Resveratrol reduces oxidative stress and apoptosis in podocytes via Sir2-related enzymes, Sirtuins1 (SIRT1)/peroxisome proliferator-activated receptor gamma co-activator 1alpha (PGC-1alpha) axis. Med Sci Monit. 2019 Feb 15;25:1220-31.

11 Yuan Y, Huang S, Wang W, Wang Y, Zhang $\mathrm{P}, \mathrm{Zhu} \mathrm{C}$, et al. Activation of peroxisome proliferator-activated receptor- $\gamma$ coactivator $1 \alpha$ ameliorates mitochondrial dysfunction and protects podocytes from aldosterone-induced injury. Kidney Int. 2012 Oct;82(7):771-89.

12 Chen J, Chen JK, Harris RC. EGF receptor deletion in podocytes attenuates diabetic nephropathy. J Am Soc Nephrol. 2015 May; 26(5):1115-25.

13 Chuang PY, Cai W, Li X, Fang L, Xu J, Yacoub $\mathrm{R}$, et al. Reduction in podocyte SIRT1 accelerates kidney injury in aging mice. Am J Physiol Renal Physiol. 2017 Sep 1;313(3):F621F28.

14 Wen D, Huang X, Zhang M, Zhang L, Chen J, $\mathrm{Gu} \mathrm{Y}$, et al. Resveratrol attenuates diabetic nephropathy via modulating angiogenesis. PloS One. 2013;8(12):e82336.

15 Kume S, Koya D. Autophagy: a novel therapeutic target for diabetic nephropathy. Diabetes Metab J. 2015 Dec;39(6):451-60.

16 Di Sante G, Pestell TG, Casimiro MC, Bisetto S, Powell MJ, Lisanti MP, et al. Loss of Sirt1 promotes prostatic intraepithelial neoplasia, reduces mitophagy, and delays PARK2 translocation to mitochondria. Am J Pathol. 2015 Jan;185(1):266-79.
17 Hasegawa K, Wakino S, Simic P, Sakamaki Y, Minakuchi H, Fujimura K, et al. Renal tubular Sirt1 attenuates diabetic albuminuria by epigenetically suppressing Claudin-1 overexpression in podocytes. Nat Med. 2013 Nov; 19(11):1496-504.

18 Li G, Chen Z, Bhat OM, Zhang Q, Abais-Battad JM, Conley SM, et al. NLRP3 inflammasome as a novel target for docosahexaenoic acid metabolites to abrogate glomerular injury. J Lipid Res. 2017 Jun;58(6):1080-90.

19 Zhang Q, Conley SM, Li G, Yuan X, Li P-L. Rac1 GTPase Inhibition blocked podocyte injury and glomerular sclerosis during hyperhomocysteinemia via suppression of nucleotidebinding oligomerization domain-like receptor containing pyrin domain 3 inflammasome activation. Kidney Blood Press Res. 2019;44(4): 513-32.

20 Conley SM, Abais JM, Boini KM, Li PL. Inflammasome activation in chronic glomerular diseases. Curr Drug Targets. 2017;18(9): 1019-29.

21 Li Y, Wang P, Yang X, Wang W, Zhang J, He $Y$, et al. SIRT1 inhibits inflammatory response partly through regulation of NLRP3 inflammasome in vascular endothelial cells. Mol Immunol. 2016 Sep;77:148-56.

22 Li Y, Yang X, He Y, Wang W, Zhang J, Zhang W, et al. Negative regulation of NLRP3 inflammasome by SIRT1 in vascular endothelial cells. Immunobiology. 2017 Mar;222(3): 552-61.

$23 \mathrm{Ma}$ CH, Kang LL, Ren HM, Zhang DM, Kong LD. Simiao pill ameliorates renal glomerular injury via increasing Sirt1 expression and suppressing NF- $\kappa \mathrm{B} / \mathrm{NLRP} 3$ inflammasome activation in high fructose-fed rats. J Ethnopharmacol. 2015 Aug 22;172:108-17. 